\title{
567 ISOFORM SPECIFIC ANTI-TGF $\beta$ THERAPY ENHANCES ANTITUMOR EFFICACY IN MOUSE MODELS OF STROMA POOR CANCERS
}

${ }^{1}$ Sadna Budhu*, ${ }^{2}$ Aditi Gupta, ${ }^{2}$ Kelly Fitzgerald, ${ }^{2}$ Rachel Giese, ${ }^{2}$ Adam Michel, ${ }^{2}$ Aliya Holland, ${ }^{2}$ Luis Felipe Campesato, ${ }^{3}$ Jacques Van Snick, ${ }^{3}$ Catherine Uyttenhove, ${ }^{3}$ Gerd Ritter, ${ }^{2}$ Jedd Wolchok, ${ }^{2}$ Taha Merghoub. 'Memorial Sloan-Kettering Cancer Center, New York, NY, USA; ${ }^{2}$ Memorial Sloan Kettering Cancer Center, New York, NY, USA; ${ }^{3}$ Ludwig Institute for Cancer Research, Brussels, Belgium

Background TGF $\beta$ is a potential target in cancer treatment due to its dual role in tumorigenesis and homeostasis. There are three isoforms of TGF $\beta$ (TGF $\beta 1$, TGF $\beta 2$ and TGF $\beta 3$ ), which are secreted by immune and non-immune cells as an inactive latent complex. Depending on the local context and players, TGF $\beta$ can adopt opposing roles in carcinogenesis and in modulating the immune system. However, the expression of TGF $\beta$ and its inhibition within the tumor microenvironment has mainly been investigated in stroma-rich tumors.

Methods We examined expression of TGF $\beta 1$ and TGF $\beta 3$ isoforms on immune cells in two stroma-poor mouse tumor models (B16 melanoma and CT26 colon carcinoma) and investigated the anti-tumor efficacy of antibodies that block TGF $\beta 1$ and TGF $\beta 3$ in these two models.

Results Depending on local expression of TGF $\beta$ isoforms, specific inhibition of either TGF $\beta 1$ or TGF $\beta 3$ may be effective. The "TGF $\beta$ signature" of CT26 colon carcinoma is defined by TGF $\beta 1$ expression on immune cells and TGF $\beta 1$ inhibition results in tumor delay; B16 melanoma has equal expression of both TGF $\beta 1$ or TGF $\beta 3$ isoforms and inhibition of either TGF $\beta 1$ or TGF $\beta 3$ controls tumor growth. We show that the mechanism of tumor growth delay is enhanced CD8 $+\mathrm{T}$ cell activation and effector function. In addition, we found that combining TGF $\beta$ inhibition with immune checkpoint blockade results in improved tumor control and survival.

Conclusions Our findings suggests that expression of TGF $\beta$ isoforms in the TME is variable in different tumor types and their expression may be used to predict anti-tumor responses to TGF $\beta$ inhibition. Isoform specific TGF $\beta$ inhibition in stroma poor tumors shifts the local immune environment to favor tumor regression alone or in combination with immune checkpoint blockade.

http://dx.doi.org/10.1136/jitc-2021-SITC2021.567 